\title{
Análisis estructural estático y modal del ala del Sistema Aéreo no tripulado "Tlapixki”" para monitoreo volcánico en México
}

\section{Static and modal structural analysis of the wing of the unmanned aerial system "Tlapixki" for volcanic monitoring in Mexico}

DOI: $10.46932 / \mathrm{sfjdv2n5-103}$

Received in: Oct 1st, 2021

Accepted in: Dec 30th, 2021

\section{Eduardo Antonio Medina Bejarano \\ Maestro en Ingeniería Civil}

Sección de Estudios de Posgrado e Investigación de ESIA Zacatenco del Instituto Politécnico Nacional Av. Luis Enrique Erro S/N Unidad Profesional Adolfo López Mateos, Zacatenco, Alcaldía Gustavo A.

Madero, CDMX.

C.P.: 07738

E-mail: medina_bejarano@hotmail.com

José Félix Vázquez Flores

Doctor en Ciencias con especialidad en Ingeniería Mecánica

ESIME U. P. Ticoman del Instituto Politécnico Nacional, México

Ticoman \#600, Col. San José Ticoman, Alcaldía Gustavo A. Madero, CDMX

C.P.: 07340

E-mail: bemol5808@gmail.com

\section{Miguel Angel Rosales Ochoa}

Maestro en Ingeniería Civil

Sección de Estudios de Posgrado e Investigación de ESIA Zacatenco del Instituto Politécnico Nacional Calle Progreso \#531, Col. Americana

Guadalajara, Jalisco

Santa María Tequepexpan,

San Pedro Tlaquepaque, Jalisco, México.

C.P.: 44160

E-mail: rosalesmiguel.lan@gmail.com

\begin{abstract}
RESUMEN
El presente trabajo consiste en el análisis Estático y modal del ala del UAS del proyecto "Tlapixki" [1] [2] que se está desarrollando en la ESIME Ticoman del Instituto Politécnico Nacional, cuya misión es apoyar al Centro Nacional de Prevención de Desastres (CENAPRED) en el monitoreo volcánico mediante toma de fotografía, video y toma de muestras de ceniza. El material utilizado en la estructura es el aluminio 2024-T3. Para el Análisis estructural se calcularon las condiciones de crucero, ascenso y descenso utilizando un paquete de elementos finitos obteniendo el estado de esfuerzos, deformaciones y sus modos de vibración, apegados a las indicaciones dadas por el Apéndice A del FAR23. Con respecto a los resultados obtenidos de la investigación, muestran que la estructura del ala analizada es capaz de soportar las cargas estructurales además de cumplir con el factor de seguridad establecido en el FAR.
\end{abstract}

Palabras claves: Avión no tripulado, Monitoreo volcánico, Análisis estructural, Prevención. 


\begin{abstract}
The present work consists in the Structural and modal analysis of the UAS wing of the "Tlapixki" [1] [2] Project that is being developed in the ESIME Ticoman of the Instituto Politécnico Nacional, whose mission is to support the National Center for Disaster Prevention (CENAPRED) in volcanic monitoring by taking photography, video of the volcano crater, and ashes samples. The material used in the structure is 2024-T3 aluminum alloy. For the Structural Analysis, the conditions of cruise, ascent and diving were calculated using a software specialized in the finite element method obtaining the stress distribution, deformations and their shape modes, attached to the indications given by the Appendix A of the FAR23. The results obtained from the research shown that the structure of the analyzed wing is capable of supporting the structural loads in addition to complying with the safety factor established in the FAR.
\end{abstract}

Keywords: Unmanned aerial system, Volcano’s Monitoring, Structural Analysis, Prevention.

\title{
1 INTRODUCCIÓN AL PROBLEMA
}

México es un país con una importante actividad volcánica, ya que cuenta con aproximadamente 3,000 volcanes, de los cuales 12 son considerados activos [3]. Uno de los volcanes que representa un gran riesgo, es el volcán Popocatépetl, debido a que se encuentran muy cercana la Ciudad de México una de las ciudades más grandes del mundo, por lo que el Centro Nacional de Prevención de Desastres (CENAPRED); por esta razón, el CENAPRED en conjunto con otras instituciones y organismos vigilan la actividad del Popocatépetl a través de una red de monitoreo, desafortunadamente esta red no dispone de una aeronave propia para realizar esta importante tarea.

\section{IMPORTANCIA DEL PROBLEMA}

El problema que enfrenta la vulcanología, es que no es posible observar el interior de los volcanes; sin embargo, aplica métodos como el registro de actividad sísmica, muestreo y análisis de ceniza, mediciones de las deformaciones del suelo y el monitoreo de gases, con el fin de evaluar el nivel de riesgo de un volcán activo.

El uso de UAVs para la recolección de datos es una alternativa, en donde no se expone persona alguna. De acuerdo al CENAPRED, ha habido intentos de sobrevolar el cráter del Popocatépetl con aeronaves no tripuladas, sin éxito hasta el momento. Por otro lado, cabe mencionar, que el costo de las aeronaves capaces de hacerlo es elevado, por lo que por lo que el proyecto que se está desarrollando en la ESIME Ticoman del Instituto Politécnico Nacional consiste en diseñar y construir un UAS, con la capacidad de volar a una altitud de 7000 m SMNM y poder realizar el monitoreo y vigilancia de la actividad volcánica en México, tomando fotografía y video del cráter, además de la toma de muestras de ceniza reduciendo costos de operación, además no comprometer la integridad de las personas que realizan dicha labor, debido a lo anterior, en este trabajo se realizaron los análisis estructurales estático y dinámico 
del ala de la aeronave Tlapixki, para asegurar que la aeronave pueda realizar dicha misión sin comprometer la integridad estructural de la misma.

\section{METODOLOGÍA}

La metodología utilizada para el modelado de la estructura de la aeronave por medio del método de los elementos finitos es la siguiente (figura 1) [4][5]:

Figura 1. Metodología utilizada en la resolución del Análisis

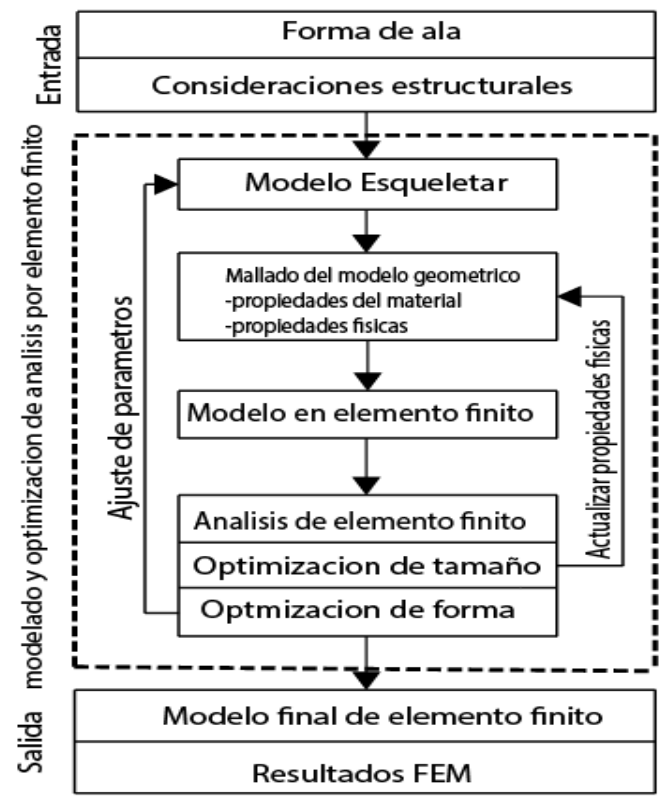

\subsection{ENTRADA}

Tomando como referencia el análisis aerodinámico previo, se propuso que la geometría del ala propuesta es de forma rectangular con una longitud de $3.30 \mathrm{~m}$, sin torcimiento geométrico ni aerodinámico, con el propósito de simplificar el análisis y manufactura del mismo. Cabe mencionar que por simetría solo se modelara el ala derecha de la aeronave para realizar el análisis.

\subsection{MODELADO Y OPTIMIZACIÓN DEL ANÁLISIS POR ELEMENTO FINITO}

El modelo estructural del ala derecha está compuesto por dos vigas (principal y secundaria), ambas con sección I constante a lo largo de toda su longitud, una piel y por 10 costillas equidistantes entre sí, el material utilizado es el aluminio 2024 T-3 debido a sus propiedades mecánicas, peso y costo. 
TABLA 1. Propiedades del Aluminio 2024 T-3 [6]

\begin{tabular}{|l|l|l|l|l|l|l|l|}
\hline \multirow{2}{*}{ Material } & $\begin{array}{c}\text { Densidad } \\
\mathrm{kg}\end{array}$ & $\begin{array}{l}\text { Esfuerzo } \\
\text { último } \sigma_{y} \\
\mathrm{mPa}\end{array}$ & $\begin{array}{l}\text { Modulo de } \\
\text { Elasticidad } \\
\mathrm{GPa}\end{array}$ & $\begin{array}{l}\text { Modulo de } \\
\text { Corte } \\
G P a\end{array}$ & $\begin{array}{l}\text { Relación } \\
\text { de Poisson } \\
v\end{array}$ & $\begin{array}{l}\text { Límite } \\
\text { Térmico } \\
{ }^{\circ} \mathrm{C}\end{array}$ & $\begin{array}{c}\text { Costo } \\
\$\end{array}$ \\
\hline $\mathrm{Al} 2024 \mathrm{~T} 3$ & 2780 & 510 & 72.4 & 28 & 0.33 & 280 & 3 \\
\hline
\end{tabular}

De acuerdo con la geometría y las propiedades de los componentes se decidió modelar las dos vigas y las costillas con elementos sólidos y la piel con un elemento Shell. Para su mallado se utilizaron elementos tetraédricos figura 3 debido a que modelan las curvas del perfil aerodinámico del ala sin crear mucha distorsión en la malla a diferencia de los elementos hexaédricos que hacían una distorsión de los elementos en el modelado de las costillas como se muestra en la figura 2 y 3.

Figure 2. Modelado de costillas elementos hexaédricos

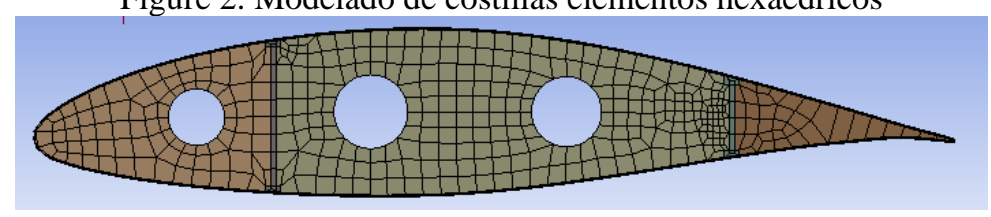

Figure 3. Modelado de costillas elementos tetraédricos

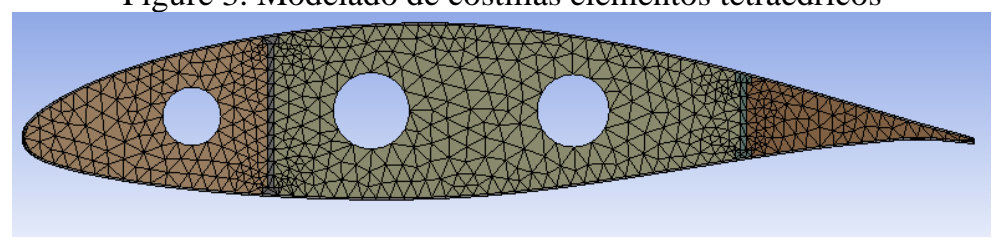

Las condiciones de frontera aplicada fue empotrar el ala de la raíz, restringiendo así los 6 grados de libertad en esa zona como se muestra en la figura 4.

Figure 4. Condiciones de frontera

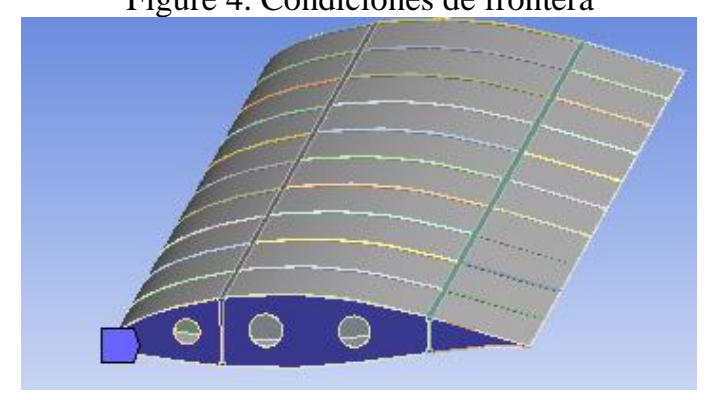

Para el cálculo de las fuerzas aplicadas en el ala en sus diferentes condiciones de vuelo se hizo uso de la envolvente de vuelo la cual es una representación gráfica de las velocidades características del avión (Crucero, ascenso, desplome etc.) y los factores de carga a los que se somete una aeronave bajo condiciones de vuelo por maniobra y por ráfaga. Representa la unión entre el análisis aerodinámico con el estructural. De la envolvente obtenida en el UAS “Tlapixki” descartamos las condiciones de ráfaga 
puesto que las líneas de ráfaga quedan dentro de la envolvente por maniobra, por lo cual nuestro avión es crítico por maniobra y no por ráfaga (figura 5).

Figure 5. Envolvente de Vuelo del UAS Tlapixki

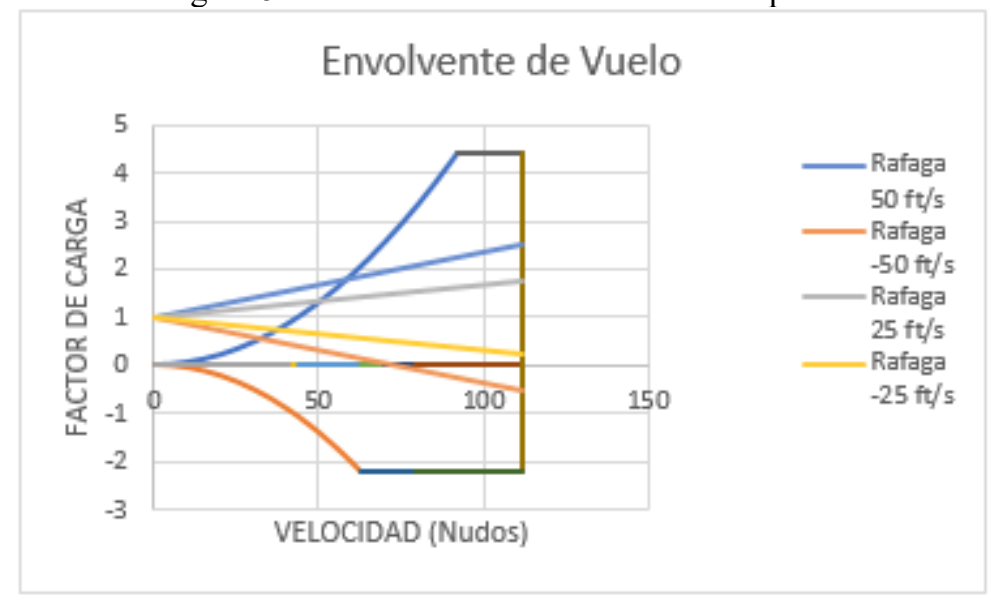

Utilizando los factores de carga obtenidos de la envolvente de vuelo los cuales son 4.4 para maniobras con ángulo de ataque positivas, y 2.2 para maniobras con ángulo de ataque negativas, además descartando las condiciones de ráfaga se calculan las diferentes condiciones de vuelo de la aeronave las cuales son:

- Alto ángulo de ataque positivo (7 grados)

- Alto ángulo de ataque negativo(-16 grados)

- Crucero (0 grados)

\section{RESULTADOS Y DISCUSIÓN}

\subsection{CRUCERO}

Se realizó un análisis estático con la interacción fluido-estructura para lo cual primero se calculó la distribución de presiones y velocidades sobre el ala cuando interactúan con el fluido en este caso el viento. Por lo cual se modeló un volumen de control haciendo un encierre a nuestra geometría en forma de un prisma rectangular.

Para poder modelar el empotre en la raíz del ala, dicha ala se aproxima a una pared del volumen de control lo suficiente, en este caso $1 \mathrm{~mm}$ [7] (figura 6 y 7). 
Figure 6. Modelado del ala y volumen de control

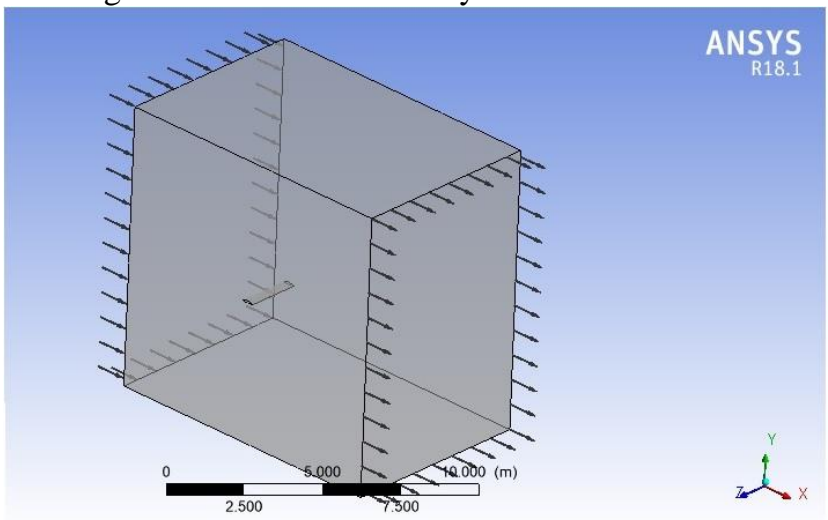

La velocidad del viento utilizada en el análisis fue la velocidad en la condición de crucero de la aeronave la cual es de $40.83 \mathrm{~m} / \mathrm{s}$, además de la densidad del aire a la altitud de 6,500 metros sobre el nivel medio del mar, la cual es de $0.626 \mathrm{~kg} / \mathrm{m}^{3}$.

Figure 7. Mallado del volumen de control

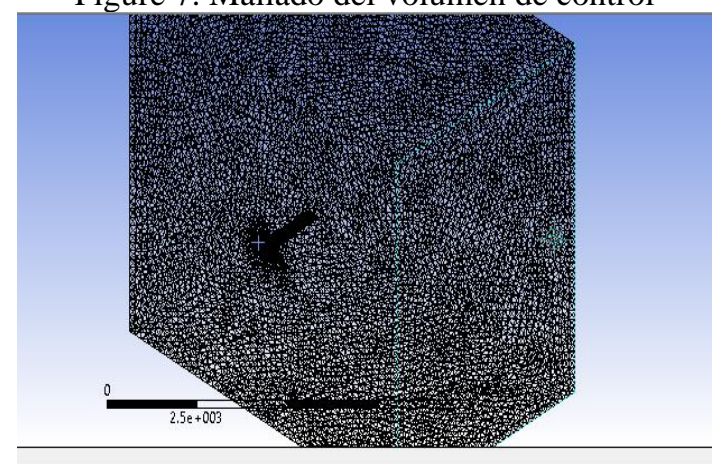

Figure 8. Distribución de presiones sobre un contorno del ala

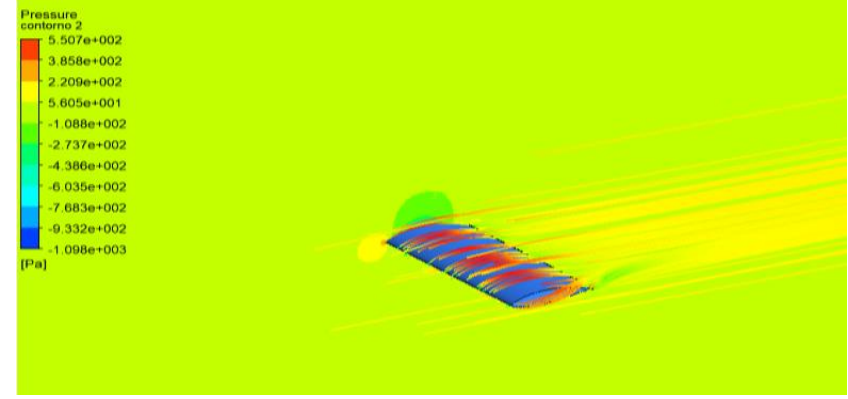

Del análisis aerodinámico hecho sobre el ala, se importan las cargas de presión estática como se muestran en la figura 8 y9 sobre la superficie de la misma [8]. 
Figure 9. Cargas de presión sobre el ala

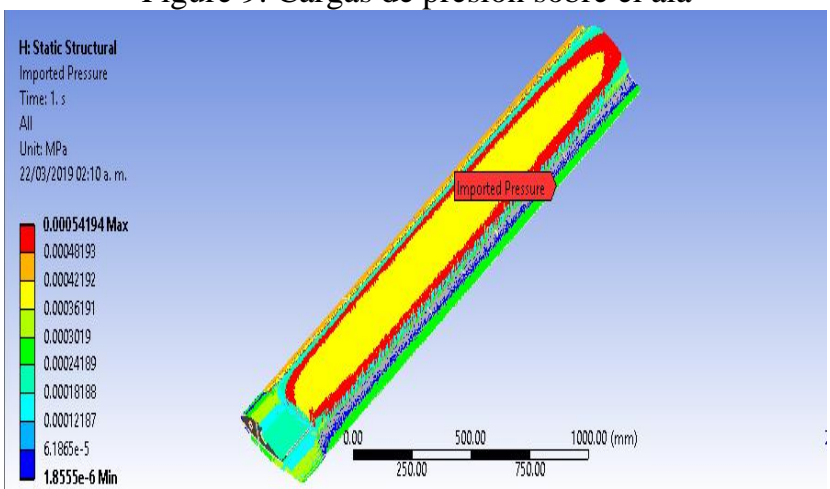

Los resultados del análisis estático por la influencia del viento nos muestran que hay una deflexión máxima en la punta del ala la cual es de $0.37829 \mathrm{~mm}$ (figura 10), la cual es muy poco significativa para la misión de la aeronave por lo cual no genera peligro alguno a la aeronave en su operación.

Figure 10. Deflexión del ala en crucero

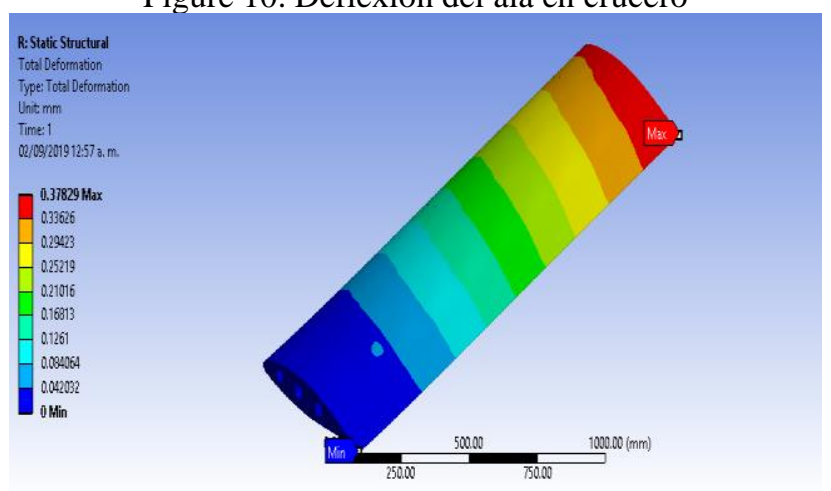

Además, la distribución de esfuerzos del ala nos muestra un esfuerzo máximo de $17.411 \mathrm{MPa}$ (figura 11), el cual se encuentra muy por debajo del esfuerzo de fluencia del material que es de $270 \mathrm{MPa}$ por lo cual cumpliría además con el factor de seguridad de 1.5 establecido en el FAR 23.

Figure 11. Distribución de esfuerzos sobre el ala en crucero

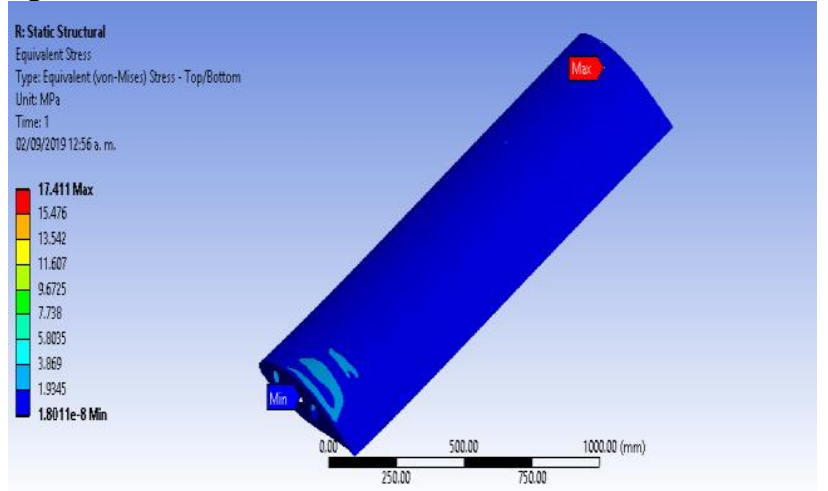




\section{Alto ángulo de ataque positivo $7^{\circ}$}

Se repite el proceso anterior, variando el ángulo de ataque del ala a 7 grados (figura 12), además cambiando la velocidad a la velocidad de maniobra de $40.83 \mathrm{~m} / \mathrm{s}$, para poder obtener las cargas de viento en dicha condición, además de considerarse en dicho análisis la fuerza y el momento producido por el empenaje vertical sobre la aeronave, dicha fuerza y momento están aplicados sobre la viga principal de la aeronave a una distancia de $0.33 \mathrm{~m}$. con una magnitud de $173 \mathrm{~N}$ y de $200 \mathrm{~N}^{*} \mathrm{~m}$. respectivamente.

Figure 12. Ala con ángulo de ataque de 7 grados

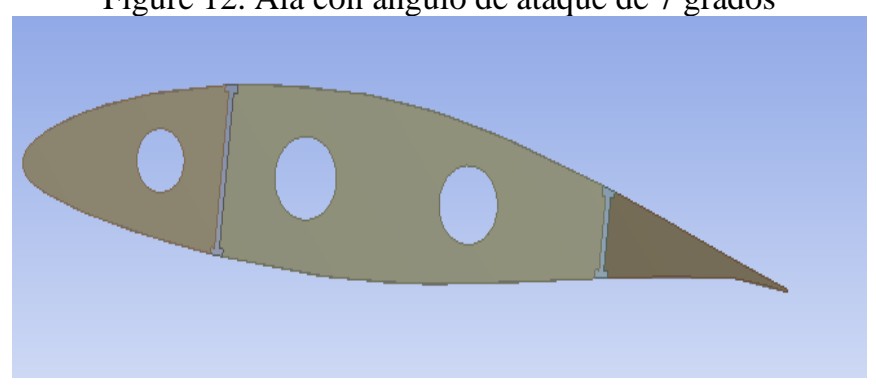

El estado de esfuerzos de esta condición se muestra en la figura 13, la cual nos muestra un esfuerzo máximo de 95.66 MPa, siendo la zona más afectada la zona más cercana la raíz del ala, comparando dicho esfuerzo con el esfuerzo con el esfuerzo de fluencia del material el cual es de $270 \mathrm{MPa}$, nos da como resultado un factor de seguridad de 2.82 cumpliendo con el factor de seguridad impuesto por el FAR 23, el cual es de 1.5.

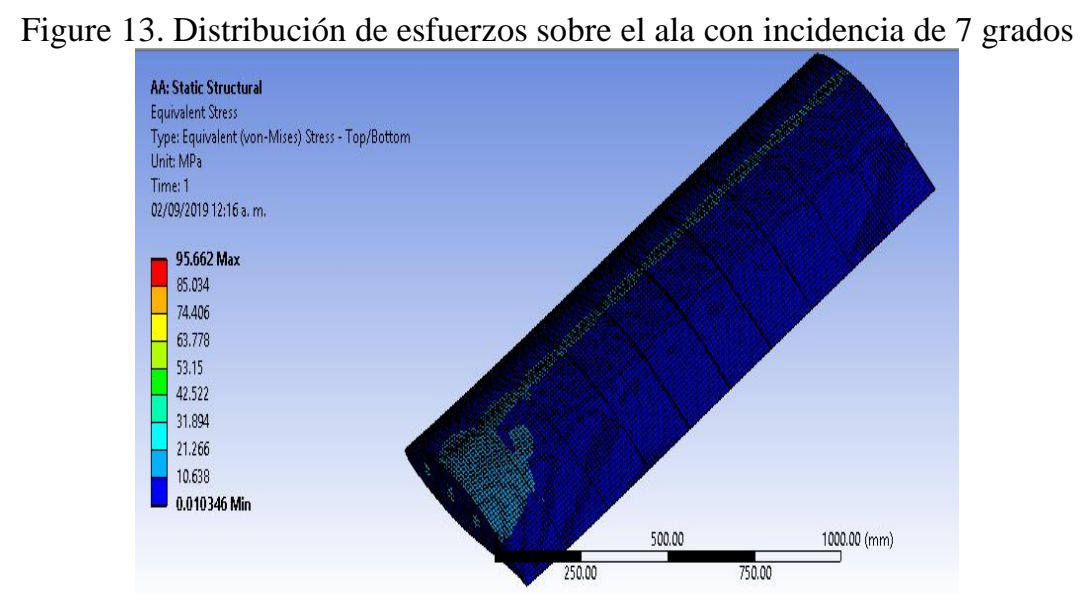

En esta condición los resultados nos muestran una deflexión máxima localizada en la punta del ala de la aeronave con un valor de $2.513 \mathrm{~mm}$, además de mostrar una configuración deformada con torsión (figura 14). 
Figure 14. Deflexión del ala con incidencia de 7 grados

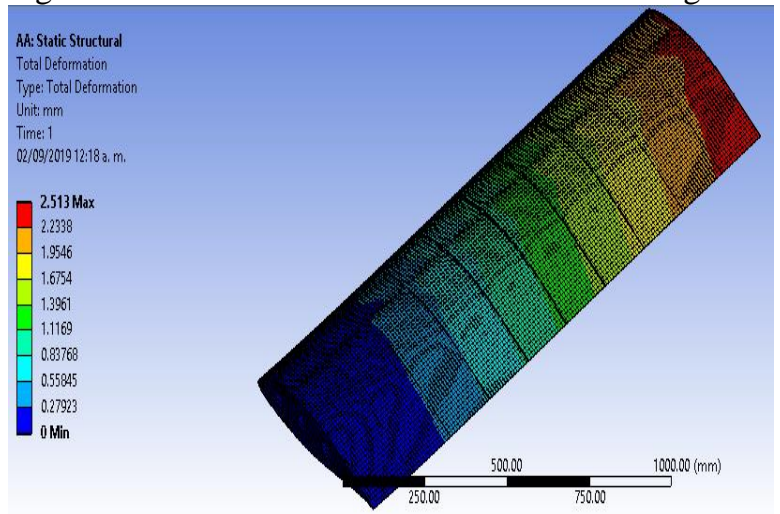

\section{Alto ángulo de ataque negativo $-16^{\circ}$}

Análogamente se repite el proceso anterior variando el ángulo de ataque del ala a -16 grados (figura 15), cambiando la velocidad del ala velocidad de descenso de $57.64 \mathrm{~m} / \mathrm{s}$, además de considerarse la fuerza y el momento producido por el empenaje vertical sobre la aeronave en dicha condición.

Figure 15. Ala en condición de descenso a -16 grados

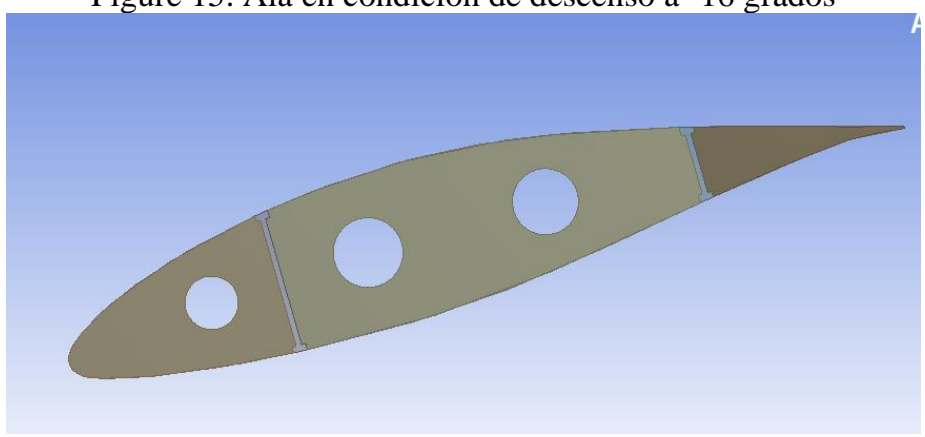

El estado de esfuerzos nos muestra un esfuerzo máximo de $132.14 \mathrm{MPa}$ (figura 16), siendo la zona más afectada la zona más cercana la raíz del ala, comparando dicho esfuerzo con el esfuerzo con el esfuerzo de fluencia del material el cual es de $270 \mathrm{MPa}$, nos da como resultado un factor de seguridad de 2.19, cumpliendo con el factor de seguridad impuesto por el FAR 23.

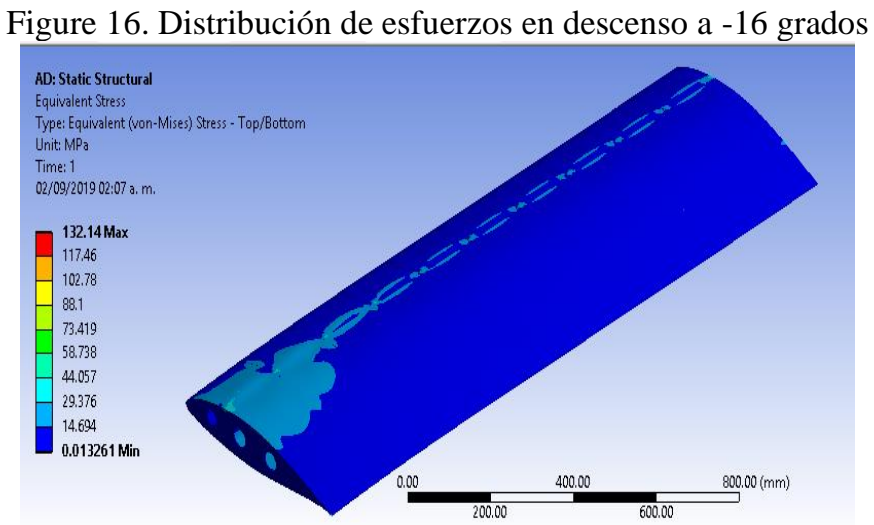


En esta condición los resultados nos muestran una deflexión máxima localizada en la punta del ala de la aeronave con un valor de 3.49 mm (figura 17), además de mostrar una configuración deformada con torsión.

Figure 17. Deflexión en descenso a -16 grados

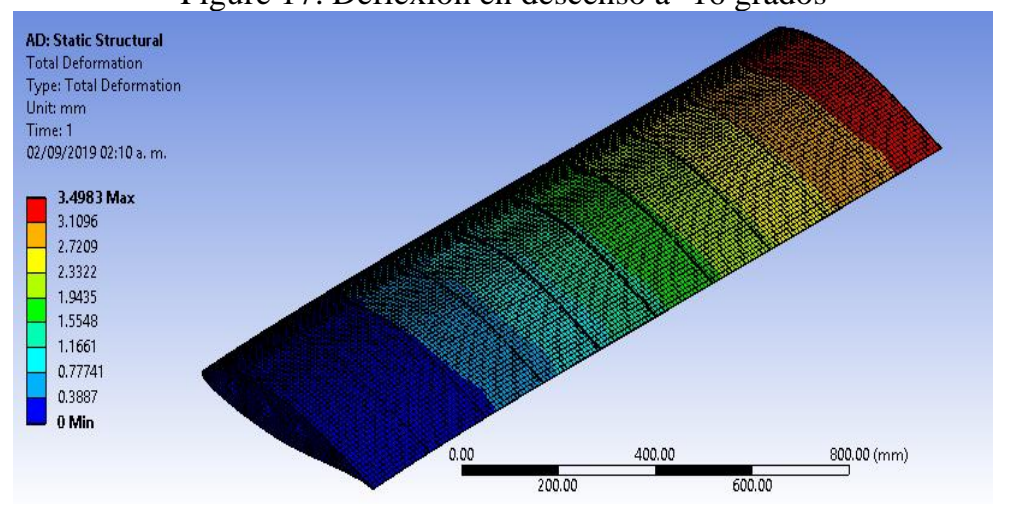

Para el análisis modal se obtuvieron 5 modos de vibración y sus frecuencias naturales asociadas [9] las cuales se muestran a continuación.

TABLA 3. Resultados del análisis modal

\begin{tabular}{|l|l|l|}
\hline Modo & Frecuencia (Hz) & Periodo (s) \\
\hline 1 & 39.46 & 0.0253 \\
\hline 2 & 117.27 & 0.0085 \\
\hline 3 & 173.67 & 0.0058 \\
\hline 4 & 231.91 & 0.0043 \\
\hline 5 & 383.41 & 0.0026 \\
\hline
\end{tabular}

El primer modo de vibración muestra una configuración solamente a flexión sobre el eje x (figura $18)$.

Figure 18. Primer modo de Vibración

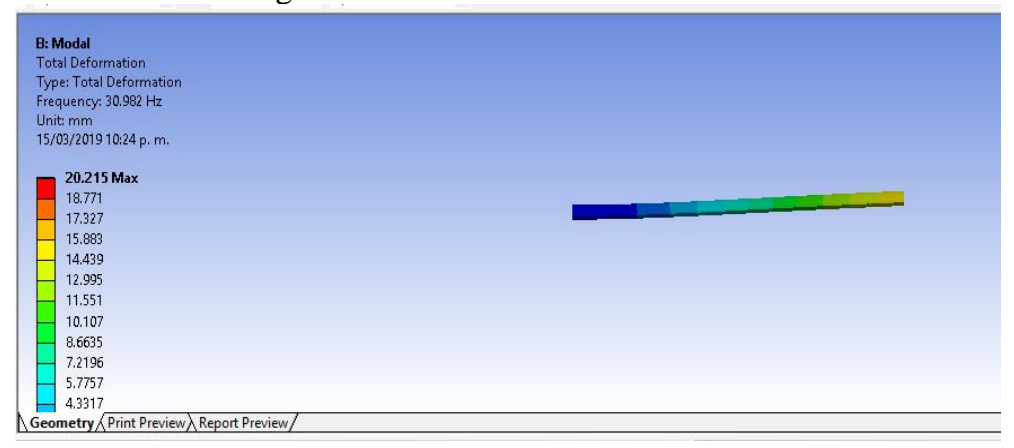

El segundo modo de vibración muestra una configuración en Flexión sobre el eje y (figura 19). 
Figure 19. Segundo modo de vibración

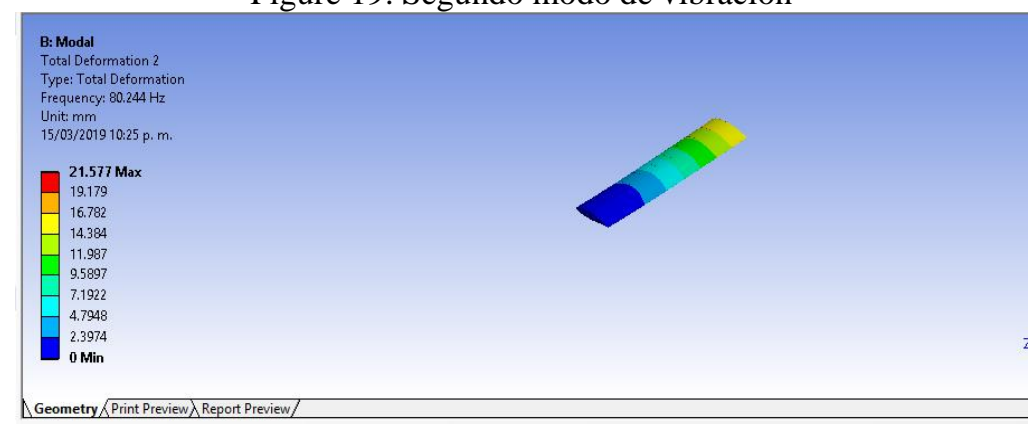

El tercer modo de vibración muestra una configuración a flexión sobre el eje x, solo que ahora mostrando más ondulaciones sobre la longitud del ala (figura 20).

Figure 20. Tercer modo de vibración

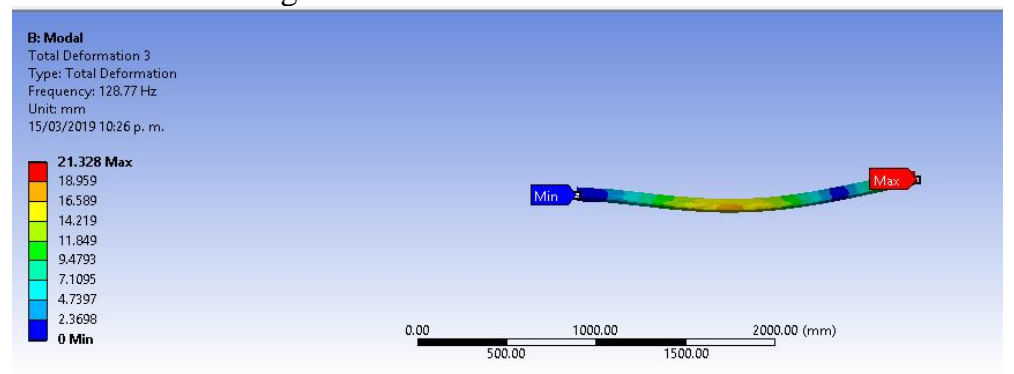

El cuarto modo de vibración ya muestra una respuesta en torsión del ala, además de mostrar modos superiores sobre los paneles del borde de salida del ala (figura 21).

Figure 21. Cuarto modo de vibración

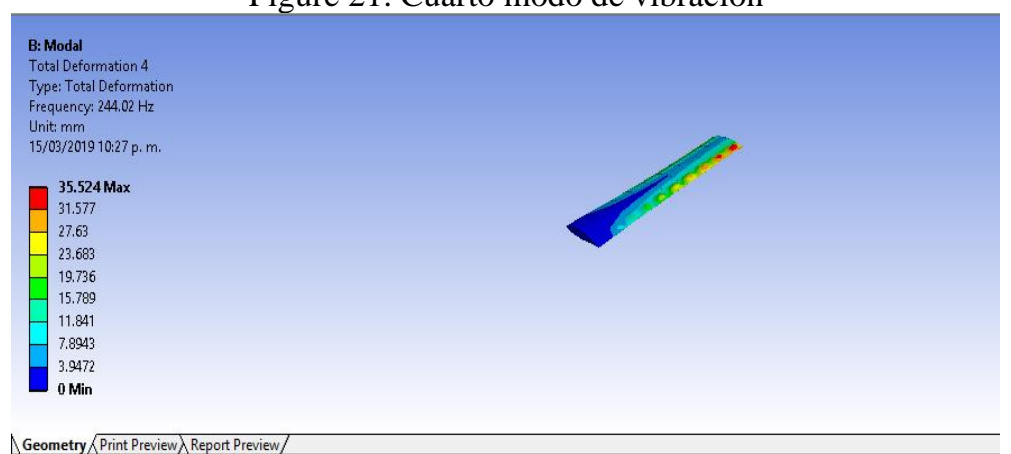

El quinto modo de vibración vuelve a mostrar flexión sobre el eje $\mathrm{x}$, aumentando el número de ondulaciones, además de seguir mostrando los modos superiores en los paneles del borde de salida (figura 22). 
Figure 22. Quinto modo de vibración

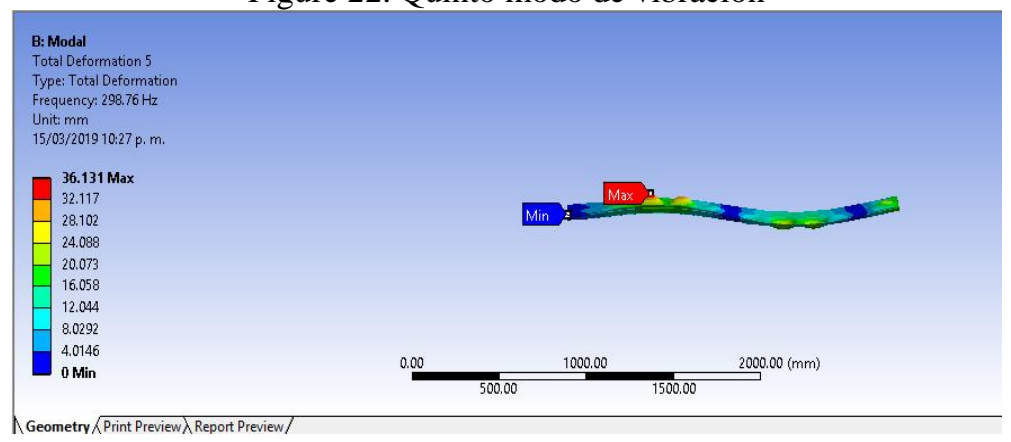

\section{CONCLUSIÓN}

Los resultados obtenidos en este análisis corresponden solamente al modelado numérico de la estructura, para poder tener certeza de los resultados obtenidos por medio del método de los elementos finitos, se debe hacer una validación mediante análisis experimental sobre el modelo real, además, se concluye que, bajo las condiciones de carga encontradas, la condición más crítica de la aeronave es con la actuación de descenso con un ángulo de -16 grados y una velocidad de $57.64 \mathrm{~m} / \mathrm{s}$, obteniendo así un esfuerzo de $132.14 \mathrm{MPa}$ y una deflexión máxima de $3.4 \mathrm{~mm}$ en la punta del ala. La estructura del ala es capaz de resistir esta actuación sin alcanzar el esfuerzo permisible del material que es $190 \mathrm{MPa}$, por lo cual cumple con lo establecido en el FAR. Mientras que, en el análisis modal, se encontraron 5 modos de vibración, los cuales generan desplazamientos muy pequeños, indicando que la estructura del ala es adecuada.

Se propone que para futuros trabajos se realice una optimización de dicha aeronave con objeto de reducir las dimensiones de los componentes estructurales sin comprometer la seguridad de estos para así hacerla más eficiente y ligera. 


\section{REFERENCIAS}

[1] Almaraz, Edgardo (2016). Diseño aerodinámico de un sistema aéreo no tripulado (UAS) para monitoreo volcánico en México. (Tesis de grado). SEPI ESIME ZACATENCO. Ciudad de México, México.

[2] Rosales, M. A. Vázquez, J.F., Correa, J.A. (oct/dic 2021). Análisis Estructural del Fuselaje de un Sistema Aéreo No Tripulado de Monitoreo Volcánico. South Florida Journal of Development.

[3] CENAPRED. Recuperado de www.cenapred.gob.mx. Accesado en Noviembre de 2015.

[4] Jiapeng, T., Ping, X., Baoyouan, Z., y Bifu, H. (2012). A finite Element parametric modeling technique of aircraft wing structures. Chinese Journal of Aeronautics 26(5), pp. 1202-1206.

[5] Manoj, K, Bhardwaj., (1997). A CFD/CSD Interaction Methodology for Aircraft Wings. Faculty of Virginia Polytechnic Institute.

[6] J. Lopes, «Material Selection for Aeronautical Structural Application, Ciência \& Tecnologia dos Materiais, vol. 20, $\mathrm{n}^{\circ}$ 3/4, pp. 78-82, 2008.

[7] Matías, Adelaido. (2014).El Método de Elemento Finito mediante El software ANSYSWB II. $1^{a}$. Ed., Ciudad de México, México: Instituto Politécnico Nacional.

[8] Filippi M., Pagani A., Carrera E., Petrolo M., Zappino E. (2012). "Advanced models for static and dynamic analysis of wing and fuselage structures". In: 8th Pegasus-AIAA Student Conference, Poitiers, France, 11-13 Abril, 2012.

[9] Vinoth, T., Waseem, A., Pabithra, M. y Srilekha, V. (2015). Static and dynamic analysis of a typical aircraft wing structure using MSC NASTRAN. International journal of research in aeronautical and mechanical engineering. 3(8), pp.1-12. 